\title{
Das Spurendokumentations-System der Polizei
}

\section{Der Gegenstand der Untersuchung}

Im Juni 198 r bildet die Schutzpolizei in Göttingen eine Spezialeinheit, das sog. Aufklärungs- und Festnahmekommando. Heute wird dies damit gerechtfertigt, daß damals zahlreiche Hausbesetzungen und behauptete Anschlußkriminalität, gemeint sind offenbar Wandbeschmierungen, Fensterscheibeneinwürfe u. ä., zu erheblicher Störung der öffentlichen Ordnung führten.' Es sei deshalb erforderlich gewesen, sich der Kleingruppentaktik des Gegenübers anzupassen und mit einer umfangreichen, i. d. R. offenen Observation die sogenannte Hausbesetzerszene aus der Anonymität zu holen und die Polizei, die bisher immer nur habe reagieren können, in die Offensive zu bringen. ${ }^{2}$ Die Polizei rühmt sich: „Ne gute Repression ist immer 'ne gute Prävention «. ${ }^{3}$

Die Arbeitsweise der Spezialeinheit ist schnell beschrieben:

Findet in Göttingen eine kulturelle oder politische Veranstaltung der "Szene « statt, so nimmt die Polizei die Kennzeichen der dort abgestellten Kraftfahrzeuge auf. Bei nächster Gelegenheit wird das Fahrzeug gestoppt, werden Fahrer und Mitfahrer kontrolliert. Ein andermal überprüft die Polizei auf diese Art die Besucher verschiedener Lokale, in denen sich die Angehörigen der "Szene « treffen. Schließlich werden bekannte Mitglieder der »Szene« auf dem Weg durch die Göttinger Innenstadt begleitet und bis zum jeweiligen Zielpunkt observiert. Die Polizei gewinnt so Informationen über Halter, Fahrer und Mitfahrer bekannter Kraftfahrzeuge, Bewohner und Besucher ausgewählter Wohngemeinschaften, Gäste bestimmter Lokale, Teilnehmer an politischen und kulturellen Veranstaltungen. ${ }^{4}$

Diese Daten werden dann in einem neuen Computersystem gespeichert. Die Polizei selbst bezeichnet das System als Spuren- und Dokumentationssystem;' amtlich hört es auf den Ruf »System $02{ }^{6}{ }^{6}$ Im Gegensatz zu dem auf Landesebene in Niedersachsen geführten System or oder POLAS ermöglicht das System 02 der Polizei, unter verschiedenen Sammelbegriffen Daten abzurufen. Während bei POLAS und den anderen bekannten Systemen ${ }^{7}$ Auskünfte immer die Kenntnis des Namens, Geburtsdatums der gesuchten oder überprüften Person vorausgesetzt wird, können die Daten bei der Spurendokumentationskartei (SPUDOK) auch nach Sammelbegriffen wie Straßennamen, Kfz-Zeichen, Kneipen etc. abgerufen werden. ${ }^{8}$

Die Datensammlung wird weiter durch allgemein zugängliche Daten, aber auch Daten anderer öffentlicher Stellen vervollständigt. So ist bekannt, daß die Daten von Zeugen in politischen Prozessen, von Kandidaten und Stadtratsmitgliedern alternativer Parteien, von Rechtsanwälten ${ }^{9}$ und von Mietern eines Universitätsgebäudes gespeichert werden. ${ }^{10}$

I Polizeioberrat Klaus Milde in einem Rundfunkinterview des Bremer Rundfunks vom 22. 3. 1983 .

2 Polizeidirektor Mogwitz, in: „Blick« vom 4. 3.1982.

3 Leiter der Kriminalpolizei Graser, in: Pokatzky, Können wir ihn aufmischen, in: „Die Zeit « vom 23. 7. 1982 .

4 All dies ist bekannt aus einem Mitschnitt des Polizeifunkes, der im März 1982 anonym in der Broschüre "Lauschangriff « in Göttingen veröffentlicht wird. Die Staatsanwaltschaft ermittelt deshalb wegen eines Verstoßes gegen das Fernmeldeanlagengesetz.

5 Pokatzky, a. a. O.

6 Blick rom 8.4.1982.

7 Z. B. HEPOLIS in Hessen und SPUDOK in Nordrhein-Westfalen.

8 Lauschangriff, $S$. I I $f$.

9 Lauschangriff, $\mathrm{S} .17 \mathrm{f}$. 
Der niedersächsische Innenminister Möcklinghoff rechtfertigte im Juli 1982 dieses Datenerfassungssystem SPUDOK vor dem Landtag. Er erklärte, daß dieses System seit Januar 1977 von der Polizei in Niedersachsen angewendet wurde. Nach Aussage des Ministers dient das System ausschließlich zur Verhinderung und Aufklärung schwerer Straftaten. Hierzu gehörten allerdings auch Sachbeschädigungen, Hausfriedensbruch und Widerstand gegen Vollstreckungsbeamte, wenn sie z. B. gemeinschaftlich oder fortgesetzt begangen werden. " Unbeantwortet ließ der Minister die Frage, ob dieses System auch in anderen niedersächsischen Städten eingesetzt werde. Dies muß aber unterstellt werden. Steinmüller ${ }^{12}$ machte schon 1979 darauf aufmerksam, daß bei der Datenverarbeitung im Sicherheitsbereich »weniges veröffentlicht [...] vieles widersprüchlich oder bewußt irreführend publiziert (ist)«.

\section{Zur Entwicklung der automatischen Datenverarbeitung in der Polizei}

Der Einsatz automatischer Datenverarbeitung in der polizeilichen Arbeit hat mit der Schaffung von SPUDOK einen neuen Höhepunkt erreicht. Um dies deutlich zu machen, sei daran erinnert, daß zu Beginn der sechziger Jahre zunächst nur daran gedacht war, die automatische Datenverarbeitung in der polizeilichen Verwaltung und zur Rationalisierung in der Fahndungsarbeit zu nutzen. ${ }^{13}$ Diese Entwicklung fand ihren vorläufigen Abschluß in dem Beschluß der ständigen Konferenz der Innenminister und -senatoren der Länder vom I6. 6. 1972 zum »Konzept eines gemeinsamen Informations- und Auskunftssystems « für die gesamte Polizei der Bundesrepublik mit dem Bundeskriminalamt als Zentralstelle ${ }^{\mathrm{I} 4}$ und in der Inbetriebnahme des elektronischen Informations- und Auskunftssystems für die gesamte Polizei (INPOL) in der Ausbaustufe Personenfahndung. ${ }^{15}$ Diese Personenfahndungskartei löste später praktisch das Deutsche Fahndungsbuch ab. Das Bundesinnenministerium feiert das System INPOL als »ein ebenso wirksames wie unentbehrliches Hilfsmittel der Verbrechensbekämpfung [. . . insbesondere für das polizeiliche Fahndungswesen ${ }^{16}{ }^{16}$

Diese Entwicklung verlangt die Zentralisierung polizeilicher Befugnisse beim Bundeskriminalamt, wie sie auch durch die Novellierung des Gesetzes über die Errichtung eines Bundeskriminalamtes in den Jahren 1969 und 1973 dokumentiert wird. ${ }^{17}$ In der Folgezeit wurde das Bundeskriminalamt mit seinem Präsidenten Horst $\mathrm{He}$ rold zum Förderer eines immer umfassender werdenden Computereinsatzes. Beispielhaft kann dies an der "Störerkartei « gezeigt werden. ${ }^{18}$ Eingeführt wurde sie bereits I 977 nach dem Beschluß der "Arbeitsgemeinschaft Kripo« vom I 3. 5 . 1977 mit dem Ziel, alle Personen zu erfassen, »die bei der Vorbereitung oder Durchfüh-

I0 Auf Anfrage teilt am 10. 5. 1982 der Präsident der Universität Göttingen dem AStA mit, daß der die Liste der Mieter eines Universitätsgebäudes an die Polizei gegeben hat. Der Brief ist veröffentlicht in: $\leadsto \mathrm{Ge}-$ heime Stadtpolizei«, herausgegeben von der Alternativ-Grünen-Initiativen-Liste (AGIL) Göttingen, 1982, S. 31 .

II Möcklinghoff, Drucksache des Nds. Landtags Io/ I I vom 4. 8. 1982.

12 Steinmüller, Der aufhaltsame Aufstieg des Geheimbereichs, in: Kursbuch 56, 1979, S. 169.

13 Schöffler, Zur Nutzbarmachung der elektronischen Datenverarbeitung für kriminalpolizeiliche Aufgaben, in: Kriminalistik 1963, 120; Ruwe, Stand und Entwicklung der elektronischen Datenverarbeitung in der Polizei, in: Die Polizei 1968, 373; Reuter, Fahnden und Fischen, in: Die Polizei 1965, S. 265.

14 Programm für die Innere Sicherheit in der BR Deutschland, Teil I, Juni 1972.

15 Elektronik im Dienste der Verbrechensbekämpfung, in: Innere Sicherheit 1974, Nr. 22, S. 9.

16 Erklärung des Bundesinnenministers vom 22. 3. 1978, in: Innere Sicherheit (1978) Nr. 43, S. 28.

17 BGBI I, 1717 (1969) und BGBI I, 701 (1973).

18 Sicherlich nicht zufällig wird hier der Begriff „Störer “ aus dem Polizeirecht verwendet und läßt das neue Aufgabengebiet erkennen. 
Ganz im Sinne Herolds wurde in diesem System eine Fülle von Daten angehäuft, die zumindest im Moment der Datenerhebung und im Regelfall wohl nie in irgendeinem strafrechtlichen oder polizeilichen Zusammenhang mit einer Straftat oder konkreten Gefahr für die öffentliche Sicherheit und Ordnung stehen. Hierdurch allein gewinnt das System Modellcharakter, der auch von offizieller Seite bestätigt wird. ${ }^{32}$

Die neue Qualität des Systems SPUDOK ergibt sich aber aus einem anderen Gesichtspunkt. Wurde ursprünglich die Computertechnik vor allem für die Vereinfachung polizeilicher Fahndungsarbeit genutzt und wurden zu diesem Zweck gesicherte Daten gespeichert, so wurden in der quasi zweiten Computergeneration z. B. dem System PIOS und den Sonderdateien für Störer und Wohngemeinschaften - unbewertete Daten aufgenommen. Unbewertet sind diese Daten, weil die Sicherheitsbehörden bei der Datenerhebung und Speicherung von ihnen nicht wissen, ob sie in einem Zusammenhang mit dem Zweck ihrer Erfassung - z. B. der Aufklärung einer Straftat - stehen. Rebmann und Stümper verteidigen diese Datensammlungen, wie ich gezeigt habe, deshalb ja mit der Behauptung, die Daten seien polizeilich relevant.

Die bekannten Datenerhebungen zum System SPUDOK belegen, daß eine Fülle neuer Daten erfaßt wurde. Daten, die in keinerlei Zusammenhang etwa mit einer Straftat oder einer Gefahr für die öffentliche Sicherheit und Ordnung stehen, die auch nicht geeignet sind, polizeilicher Arbeit im gesetzlichen Auftrag zu dienen. So ist beispielsweise überhaupt nicht zu erkennen, welche polizeiliche Relevanz die gespeicherte Mitgliedschaft in einem Kommunalparlament, die Tatsache haben soll, ein fremdes Auto an einem bestimmten Tag gefahren zu sein, eine kulturelle Veranstaltung besucht zu haben. Tatsächlich interessiert die Polizei an diesen Daten nicht ihr verifizierbarer Tatsacheninhalt. Interesse findet allein der hinter den Tatsachen vermutete soziale Zusammenhang. Aus der Fülle der Daten, so muß vermutet werden, soll schließlich ein Sozialdiagramm der "Szene« und sollen die sozialen Beziehungen Einzelner erkennbar werden. Offenbar sind die Sicherheitsbehörden der Auffassung, die Verknüpfung dieser Sozialdaten ließe eindeutige Schlüsse zu.

Kennzeichnend für diese Datenarbeit, und auch dies bezeichnet die neue Qualität, ist das Eindringen der Polizei in allein dem Verfassungsschutz obliegende Aufgaben oder doch die Übernahme der Arbeitsmethoden des Verfassungsschutzes in die polizeiliche Tätigkeit. Allein dem Verfassungsschutz nämlich obliegt es, im Vorfeld konkreter Gefahr zu beobachten. $\S_{3}$ Abs. 3 BVerfSchG ${ }^{33}$ ermächtigt ihn dazu. Voraussetzung ist hier allerdings, daß die Beobachtung verfassungsfeindlichen Bestrebungen gilt. Die Rechtsprechung, in Hausbesetzern auch eine kriminelle Vereinigung sehen zu können, und die Ausführungen von Rebmann und Stümper mögen jedoch die Polizei zu identischen Arbeitsmethoden angeregt haben. Jedenfalls erinnert das System SPUDOK deutlich an das System NADIS des Verfassungsschutzes. Wie der Verfassungsschutz aus dem Besuch bestimmter Veranstaltungen auf die Mitgliedschaft in einer bestimmten politischen Partei und aus ihr und dem gelegentlichen Zeitungsverkauf auf mangelnde Verfassungstreue schließen will, so wollen auch im System SPUDOK die Sicherheitsbehörden deuten.

Offenbar irritiert die Sicherheitsbehörden in dieser Praxis auch nicht die Nähe zu einem Verständnis von Polizei, wie sie es bestimmt nicht wollen. Möglicherweise ist es nur geschichtliche Blindheit, die sie in der Schaffung und dem Einsatz solcher Systeme, wie SPUDOK es ist, fortfahren läßt.

32 Polizeidirektor Mogwitz, in: Pokatzky, a. a. O.

33 Gesetz über die Zusammenarbeit des Bundes und der Länder in Angelegenheiten des Verfassungsschutzes vom 27. 9. 1950 (BGBl S. 682), geänd. durch VerfSchutz-Ändg. v. 7.8. 1972 (BGBI I, S. I 382 ). 
Schon die Übernahme nachrichtendienstlicher Mittel i.S.d. $₫ 3$ Abs. 3 S. 2

BVerfSchG in die polizeiliche Arbeit begründet den Vorwurf, die aus Erfahrung im Grundgesetz gewollte strikte Trennung von Polizei und Verfassungsschutz werde verletzt. Offenbar haben jene, die jetzt derartige Systeme fordern und fördern, vergessen, daß der Kontrollrat in seinem Gesetz Nr. 31 vom 1. Juli 1946 einer deutschen Polizei ausdrücklich verbat, die politische Betätigung von jedermann zu überwachen oder zu kontrollieren ${ }^{34}$ und die drei westlichen Militärgouverneure in ihrem Schreiben vom 14. April 1949 die Übertragung polizeilicher Befugnisse an ein Amt zum Schutz vor einem Umsturz untersagten." In dem Genehmigungsschreiben zum Grundgesetz vom 12. Mai 1949 wurde diese Trennung von Aufgabenzuweisung und Befugnis noch einmal für jede Auslegung des Grundgesetzes festgeschrieben. ${ }^{36}$

An dieser Stelle sei daran erinnert, daß auch den Nationalsozialisten die strikte Festlegung der Weimarer Polizei auf die Strafverfolgung und Gefahrenabwehr das größte Ärgernis war. Die nationalsozialistische Polizei hatte demgegenüber die Aufgabe, "soziale Zersetzungserscheinungen schon im Keime zu ersticken «. ${ }^{7}$

\section{Zur rechtlichen Beurteilung}

In der Beschreibung des Systems SPUDOK ist deutlich geworden, daß hier versucht wird, ein umfassendes Bild ausgewählter Gruppen, der persönlichen Verhältnisse des einzelnen Gruppenmitgliedes, seiner sozialen und politischen Kontakte zu gewinnen.

Diese Praxis verletzt die Grundrechte der Achtung der Menschenwürde (Art. I Abs. 1 GG) und der freien Entfaltung der Persönlichkeit (Art. 2 Abs. 1 GG). Das Bundesverfassungsgericht hat hierzu festgestellt:

»Es widerspricht der menschlichen Würde, den Menschen zum bloßen Objekt im Staate zu machen. [. . .] Mit der Menschenwürde wäre es nicht zu vereinbaren, wenn der Staat das Recht für sich in Anspruch nehmen könnte, den Menschen zwangsweise in seiner ganzen Persönlichkeit zu registrieren und zu katalogisieren, [. . .] und ihn damit wie eine Sache zu behandeln, die einer Bestandsaufnahme in jeder Beziehung zugänglich ist. « ${ }^{38}$

Natürlich würden jene, die SPUDOK und andere Systeme einführen, entrüstet behaupten, sie kennten diese Entscheidung und würden nach ihr handeln. Wenn im Wissen um diese Verteidigung das BVerfG so ausführlich zitiert wird, so hat das gleichwohl einen Sinn: Die Grundrechte ${ }^{39}$ markieren die letzten Grenzen auch polizeilichen Handelns, das sich deshalb an ihnen messen lassen muß. Vor allem aber bedarf jedes staatliche Handeln, das in grundrechtlich gesicherte Rechtspositionen eingreift, gemäß Art. 20 Abs. 3 GG einer gesetzlichen Ermächtigungsgrundlage. $4^{\circ}$

34 Amtsblatt des Kontrollrates, 1946, S. 54

35 "Polizeibrief ", abgedruckt bei Hans Schneider, Polizeirecht, I2. Aufl. München und Berlin I950, S. $436 \mathrm{f}$.

36 Abgedruckt bei Jürgen Seifert, Grundgesetz und Restauration, 3. Aufl. Darmstadt und Neuwied 1977, S. $198 \mathrm{f}$.

37 Höhn, in: Frank/Himmler/Best/Höhn, Grundlagen der deutschen Polizei, 1937, S. 26; zit. nach: Schwan, Offener Brief an den Bundesminister der Justiz, in: Demokratie und Recht 1980, S. 337.

38 BVerfGE 27, I (6); vgl. auch BVerfGE 6, S. 32.

39 Dazu ist hier nicht nur die Menschenwürde (Art. I Abs. I GG), das Persönlichkeitsrecht (Art. 2 Abs. 2 GG), sondern vor allem an das Recht der freien Meinungsäußerung (Art. s GG) und die Grundrechte der Versammlungs- (Art. $8 \mathrm{GG}$ ) und Vereinigungsfreiheit (Art. $9 \mathrm{GG}$ ) zu denken.

40 Stern, Das Staatsrecht der Bundesrepublik Deutschland, Band I, München 1977, S. 635; v. Münch, in: Erichsen/Martens, Allgemeines Verwaltungsrecht, Berlin/New York 1975, S. 40 f. - völlig erstaunlich. sind deshalb die vielen Rechtfertigungsversuche derartiger Praxis durch ministerielle Erlasse. 

die Erhebung von Daten in Grundrechte eingreift. Das BVerfG begründet dies überzeugend mit dem psychischen Druck, der den Einzelnen in seiner freien Entfaltung der Persönlichkeit hemmen könnte, wenn er erwarten müsse, in allen Lebensäußerungen erfaßt zu werden. ${ }^{41}$ Simon und Taeger ${ }^{42}$ begründen den Eingriffscharakter zutreffend damit, daß durch eine derartige Datenerhebung bzw. Speicherung die »individuelle Lebensgestaltung tendenziell eingegrenzt und auf gesellschaftlich durchschnittliche Erwartungsnormen zurechtgestutzt (wird). « Die Datenerhebung ${ }^{43}$ in Göttingen und die Speicherung der Daten in dem System SPUDOK können deshalb nur rechtmäßig sein, wenn eine gesetzliche Ermächtigungsgrundlage diese Eingriffe erlaubt.

Die Polizei selbst stützt ihre Arbeit immer wieder auf $\$ 9$ des niedersächsischen Datenschutzgesetzes. ${ }^{44}$ Diese Vorschrift jedoch spricht in ihrem Absatz I zunächst überhaupt nicht von der Datenerhebung, sondern allein von der Speicherung und Veränderung der Daten. $\$ 9 \mathrm{Abs}$. I Nds. DSG will also nach seinem Wortlaut die Datenerhebung nicht regeln und kann sie darum nicht erlauben..$^{45}$ In $\$ 9$ Abs. 2 Nds. DSG wird allerdings verlangt, daß der Betroffene auf die Rechtsgrundlage der $\mathrm{Da}$ tenerhebung hingewiesen wird. Hieraus folgt nicht nur, daß der Gesetzgeber - was mit Blick auf Art. 20 Abs. 3 GG selbstverständlich ist - eine Befugnisnorm zu jeder Datenerhebung verlangt, sondern vor allem - was früher einmal bestritten wurde -, daß der Gesetzgeber die Datenerhebung als Eingriff wertet und $\$ 9$ Nds. DSG selbst diese Berechtigung nicht gibt. ${ }^{46}$ Andernfalls hätte der Gesetzgeber verlangen können, den Betroffenen auf $\$ 9$ Nds. DSG hinzuweisen, also keine offene Formulierung wählen müssen. $\$ 9 \mathrm{Nds}$. DSG scheidet deshalb als Befugnisnorm zur Datenerhebung aus.

Die Befürworter derart umfangreicher Datenerhebungen, wie sie z. B. zum Aufbau des SPUDOK-Systems erforderlich sind, verweisen dann auf $\$ 163$ StPO. Diese Vorschrift soll als Generalklausel die Datenerhebung rechtfertigen. ${ }^{47} \mathrm{Im}$ Sinne dieser Argumentation hat das Bundesinnenministerium im 2. Datenbericht über die Dateien des Bundeskriminalamtes für den Bereich der "Beobachtenden Fahndung" festgestellt, daß ) diese Vorschriften (gemeint sind die $\$ \$ 16 \mathrm{I}, 163 \mathrm{StPO}$ ), die in erster Linie eine Aufgabenzuweisung beinhalten, dann auch als Grundlage für Rechtseingriffe herangezogen werden können, wenn es sich dabei um Maßnahmen handelt, deren Eingriffsintensität unterhalb der in der StPO besonders geregelten Eingriffe liegt $\ll 4^{4}$

$4^{1}$ BVerfGE 27, I (6); vgl. auch Schwan, Datenschutz - Vorbehalt des Gesetzes und Freiheitsgrundrechte, in: Verwaltungsarchiv i975 (2), 120 (128); Fahndung und Datenschutz, in: Recht und Politik (1980) S. 74 .

42 Simon/Taeger, Rasterfahndung, Baden-Baden 1981, S. 43; Schmidt, Die bedrohte Entscheidungsfreiheit, in: JZ r 974, 24I (245)

43 Als Datenerhebung soll mit Dammann (in: Simitis u. a., Kommentar zum Bundesdatenschutzgesetz, Baden-Baden 1978, S. 34I) jede Aktivität verstanden werden, durch die im Wege systematischer Befragung, Beobachtung oder Messung Daten aus verschiedenen Informationsquellen zusammengestellt werden, um über sie verfügen zu können.

44 Die Bestimmungen des Nds. Datenschutzgesetzes (Nds. GVBI. 1978, S. 42 I ff.) entsprechen den Bestimmungen des Bundesdatenschutzgesetzes vom 27.1. 1977 (BGBl. I, 201 ff.): "Das Speichern und das Verändern personenbezogener Daten ist zulässig, wenn es zur rechtmäßigen Erfüllung der in der $\mathrm{Zu}-$ ständigkeit der speichernden Stelle liegenden Aufgaben erforderlich ist. *

45 Dammann, a. a. O., S. $330 \mathrm{f}$.

${ }_{4} 6$ Riedel, Probleme des Datenschutzes im Bereich der polizeilichen Tätigkeit, in: Die Polizei 1978, S. 272.

47 Schwan, Die Abgrenzung des Anwendungsbereichs der Regeln des Straf- und OrdnungswidrigkeitenVerfolgungsrechts yon dem des Rechts der Gefahrenabwehr, in: VerwA (1979), 2, S. 109 (118) hat hier die sog. Schwellentheorie entwickelt.

$4^{8}$ Zitiert nach: FR vom 27. 3. 1980. 
Diese Position kann jedoch aus vielen Gründen nicht überzeugen. Zum einen muß schon zweifelhaft sein, ob die danach erlaubte Datenerhebung tatsächlich weniger intensiv in die Rechte des Einzelnen eingreift als die Maßnahmen der StPO es tun. Hier muß man vielmehr mangelnde Sensibilität vermuten, ist doch an die prinzipielle Verunsicherung des Einzelnen zu denken, wenn er gewärtig sein muß, seine privaten Lebensäußerungen könnten jederzeit erfaßt werden. Nach der Rechtsprechung des Bundesverfassungsgerichtes stehen immerhin die Würde des Menschen und sein Grundrecht der freien Entfaltung seiner Persönlichkeit auf dem Prüfstand. Zum anderen unterläuft diese Argumentation das Rechtsstaatsprinzip, das für jeden Eingriff und nicht nur für den geregelten Eingriff eine Ermächtigungsgrundlage fordert. Schließlich verkehrt diese Interpretation den gesetzgeberischen Willen in sein Gegenteil. Demnach nämlich soll $\$ 16_{3}$ StPO gerade keine Ermächtigungsgrundlage, sondern allein Aufgabenzuweisung sein.49

Im übrigen verlangt $\oint_{1} 6_{3}$ StPO in jedem Fall einen gegenüber dem Anfangsverdacht konkreteren Tatverdacht. ${ }^{\circ}$ Die Datenerhebung im System SPUDOK ist gerade dadurch gekennzeichnet, daß dieser Verdachtsgrad nicht vorliegt.

Als Befugnisnorm kommt schließlich die polizeiliche Generalklausel in Betracht. Sie verlangt, daß für die polizeiliche Maßnahme eine konkrete Gefahr vorliegt. ${ }^{51}$ Gefahr ist dabei die erkennbare, objektive, nicht entfernte Möglichkeit des Schadenseintrittes. Eine konkrete Gefahr liegt danach vor, wenn diese Voraussetzungen im konkreten Einzelfall gegeben sind..$^{2}$ In diesem Zusammenhang muß nochmals auf die Entscheidung des BVerfG verwiesen werden. Die Entscheidung des Gerichts gründet sich in dem verfassungsrechtlich verankerten Gebot der Verhältnismäßigkeit und besonders des Übermaßverbotes. ${ }^{53}$ Dammann ${ }^{54}$ stellt hierzu fest, daß »beispielsweise die Aufgabe, Gefahren für die öffentliche Sicherheit und Ordnung abzuwenden, nicht den in einer dauernden und systematischen, personenbezogenen Erfassung und Speicherung von Fahrzeugbewegungen (nach polizeilichen Kennzeichen, Zeit, Ort, Fahrtrichtung) liegenden Eingriff in das Grundrecht der Freiheit der Person (Art. 2 Abs. 2 Satz 2 GG) « rechtfertige.

Einleitend wurde die Arbeitsweise des Aufklärungs- und Festnahmekommandos dargestellt. Es ist erkennbar, daß in keinem Einzelfall eine konkrete Gefahr angenommen werden kann und regelmäßig gegen das Übermaßverbot verstoßen wird. Selbst wenn die Datenerhebung in einem - möglicherweise nicht dokumentierten Einzelfall - rechtmäßig erfolgt sein sollte, so wird in der Masse der Fälle - und erst die Summe der Daten macht das System SPUDOK - die Speicherung der Daten nicht erforderlich im Sinne des $\$ 9$ Abs. I Nds. DSG und mithin rechtswidrig gewesen sein." Die Datenspeicherung muß dabei zur Aufgabenerfüllung notwendig, nicht nur geeignet gewesen sein. Unzulässig ist so, Daten auf Verdacht, eben ohne

49 Bull, Fahndung und Datenschutz, in: Bundeskriminalamt (Hg.), Möglichkeiten und Grenzen der Fahndung, S. 57 (59).

$50 \mathrm{Simon} / \mathrm{T}$ aeger, a. a. O, S. 54 .

51 Riedel, a. a. O.; ders.: Datenschutz bei den Sicherheitsbehörden, Köln/Berlin/Bonn/München I 978 , S. 9 .

52 Götz, a. a. O., S. s6; BVerwG DVBI. 1973, S. $857(859)$.

53 Vgl. dazu Stern, a. a. O., S. $671 \mathrm{ff}$.

54 Dammann, a. a. O., S. 336

55 Damman, a. a. O., S. 337, der beispielhaft feststellt, daß es bei einer Razzia erforderlich sein kann, die Personalien der Anwesenden aufzunehmen, nicht aber die Daten Unverdächtiger zu speichern. Als der Pressesprecher der AGIL in Erinnerung an die Verhaltensweise der Geheimen Staatspolizei im Hitlerfaschismus das Göttinger Spudok-System »Geheime Stadtpolizei» betitelte, wurde gegen ihn Anklage wegen Beleidigung erhoben. In erster Instanz hat das Amtsgericht Göttingen verurteilt. Es hat sich dabei geweigert, die auch in diesem Artikel in Abschnitt 3 dargestellte Parallele zum uferlosen Gefährdungsbegriff der nationalsozialistischen Polizei zu würdigen. 

diesem Ziel scheint SPUDOK zu dienen. Aus $\$ 9$ Nds. DSG ergibt sich aber auch, daß Daten nur so lange gespeichert werden dürfen, wie eine konkrete Gefahr besteht. Eine darüber hinausgehende Speicherung ist unzulässig, weil sie nicht mehr zur Aufgabenerfüllung erforderlich ist.

\section{Ausblick}

Die hier dargestellte Idee polizeilicher Datenarbeit und die belegte Praxis der Datenerhebung können nicht ernst genug genommen werden. Wenn nämlich der Leiter der Schutzpolizei Göttingen, Polizeidirektor Mogwitz, dem System SPUDOK Modellcharakter bescheinigt, 57 so scheint dies in doppelter Sicht wahr. Zum einen gilt dies sicherlich für die räumliche Ausdehnung des Systems und den Umfang der Datenerhebung. Zum anderen aber könnte dem System SPUDOK Modellcharakter auch im Hinblick auf das Erkenntnisobjekt zukommen. Was heute gegen die "Szene" gerichtet ist, kann jederzeit auf andere Gruppen übertragen werden. Wie leicht eine solche Anwendung fällt, beweist die 1979 bekannt gewordene Überprüfung aller Kandidaten zu den Betriebsratswahlen in Bremen ${ }^{88}$. Auch hier wurde eine Arbeitsmethode genutzt, die zuvor gegen den Linksextremismus eingerichtet wurde. ${ }^{99}$ In diesem Sinn ist wahrscheinlich, daß Staat und Polizei entsprechend z. B. gegen einen umfangreichen Streik und die Beteiligten vorgehen werden. Die Innenminister als Polizeioberbehörde selbst denken offenbar an diese Möglichkeit, wenn sie im Gliederungspunkt 3.4.3.3 der Polizeidienstvorschrift bestreikte Betriebe und Versammlungslokale ihrem Schutz unterstellen. ${ }^{60}$

Christoph Lebmann

56 Bull, Entscheidungsfragen in Sachen Datenschutz, in: ZRP 1975, S. 7 (12).

57 Mogwitz, in: *Blick« vom 4. 3. 1982.

s8 "Stern« vom 17. 9. 1979.

59 Vgl. auch: Verfassungsschutzmosaik - einige Steinchen zur Aktivität des Verfassungsschutzes im betrieblichen und gewerkschaftlichen Bereich, in: CILIP 1980, Nr. 5, S. 29 m. w. N.

60 PDV 100, abgedruckt in: Arbeiterkampf Nr. 198, 1981. 